Document downloaded from:

http://hdl.handle.net/10251/123228

This paper must be cited as:

Battistoni, M.; Magnotti, GM.; Genzale, CL.; Arienti, M.; Matusik, KE.; Duke, DJ.; GiraldoValderrama, JS.... (2018). Experimental and Computational Investigation of Subcritical NearNozzle Spray Structure and Primary Atomization in the Engine Combustion Network Spray D. SAE International Journal of Fuel and Lubricants. 11(4):337-352. https://doi.org/10.4271/2018-01-0277

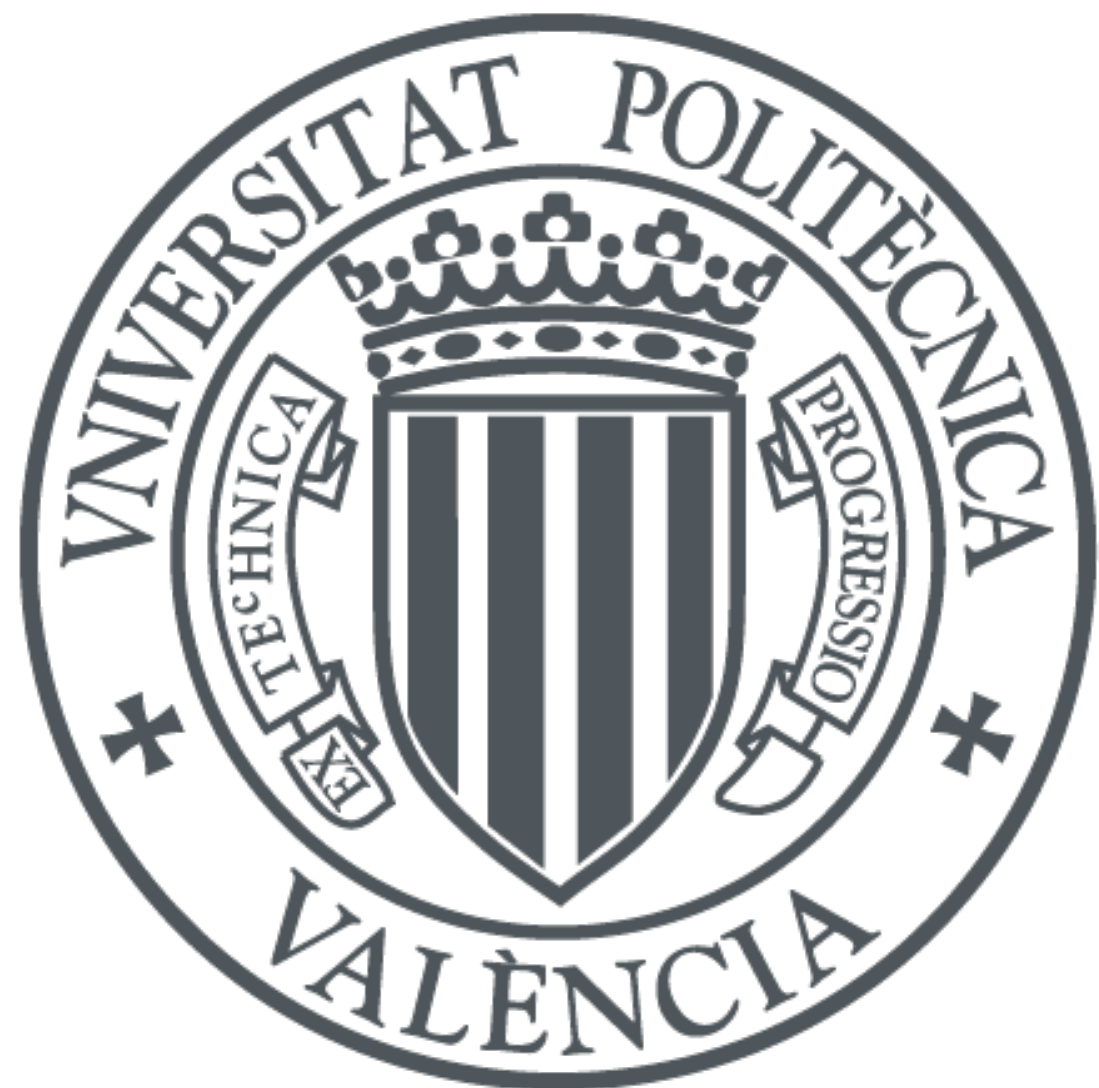

The final publication is available at

https://doi.org/10.4271/2018-01-0277

Copyright SAE International

Additional Information 


\title{
Experimental and Computational Investigation of Subcritical Near-Nozzle Spray Structure and Primary Atomization in the Engine Combustion Network Spray D
}

\author{
Author, co-author (Do NOT enter this information. It will be pulled from participant tab in \\ MyTechZone) \\ Affiliation (Do NOT enter this information. It will be pulled from participant tab in MyTechZone)
}

\begin{abstract}
In order to improve understanding of the primary atomization process for diesel-like sprays, a collaborative experimental and computational study was focused on the near-nozzle spray structure for the Engine Combustion Network Spray D single-hole injector. These results were presented at the 5th Workshop of the Engine Combustion Network in Detroit, Michigan. Application of x-ray diagnostics to the Spray D standard cold condition enabled quantification of distributions of mass, phase interfacial area, and droplet size in the near-nozzle region from 0.1 to $14 \mathrm{~mm}$ from the nozzle exit. Using these data, several modeling frameworks, from Lagrangian-Eulerian to Eulerian-Eulerian and from Reynolds-Averaged Navier Stokes (RANS) to Direct Numerical Simulation (DNS), were assessed in their ability to capture and explain experimentally observed spray details. Due to its computational efficiency, the Lagrangian-Eulerian approach was able to provide spray predictions across a broad range of conditions. In general, this "engineering-level" simulation was able to reproduce the details of the droplet size distribution throughout the spray after calibration of the spray breakup model constants against the experimental data. Complementary to this approach, higher fidelity modeling techniques were able to provide detailed insight into the experimental trends. For example, interface-capturing multiphase simulations were able to capture the experimentally observed bi-modal behavior in the transverse interfacial area distributions in the near-nozzle region. Further analysis of the spray predictions suggests that peaks in the interfacial area distribution may coincide with regions of finely atomized droplets, whereas local minima may coincide with regions of continuous liquid structures. The results from this study highlight the potential of $x$-ray diagnostics to reveal salient details of the near-nozzle spray structure, and to guide improvements to existing primary atomization modeling approaches.
\end{abstract}

\section{INTRODUCTION}

Direct injection compression ignition (CI) engines have become the preferred architecture for advanced engine concepts due to their high thermal efficiency and power output [1]. However, CI engines are known to produce high levels of NOx and particulate matter. With increasingly stringent emissions standards, advanced combustion control strategies have been extensively researched as a way to reduce the in-cylinder emissions signature [2]. In particular, partially premixed combustion (PPC) strategies advance the timing of start of injection (SOI) with respect to top dead center (TDC) to control fuelair mixing and auto-ignition processes, and thereby pollutant

Page 1 of 14 formation [3]. However, this causes a more complex link between mixture formation and combustion. Therefore, the effectiveness of such strategies can rely profoundly on the initial spray development and droplet formation processes through their influence on vaporization and fuel-air mixing.

If computational design tools are to be used to guide the use of direct injection strategies for cleaner and more fuel-efficient engines, accurate representation of the fuel spray within an engine simulation is essential. However, the spray formation process is challenging to model in the Reynolds and Weber number regimes relevant to fuel sprays [4], and for atomization processes driven not only by linear instabilities, but also by non-linear instabilities, as recently determined in $[5,6]$. In particular, the near-nozzle region is characterized by high mass loadings of the liquid phase and large momentum exchanges, therefore requiring specialized numerical techniques that are the subject of much ongoing research [7, 8]. To accurately represent the spray and subsequent droplet formation processes, care must be taken to model the evolution of the liquid-gas interface. Using an interface capturing approach, such as the advanced coupled level set and volume-of-fluid (CLSVOF) method [9], or a basic VOF method [10], the dynamics of the interface can be resolved directly. However, the extremely fine droplets generated by the spray due to the high Re and We numbers result in a tremendous burden on the direct simulation of this type of two-phase flow, where the complexity of the interfacial area rapidly necessitates very high grid densities. Alternatively, the interface dynamics can be described by assuming that the interface is diffuse, as is done in the Eulerian $\Sigma-Y$ approach [11-13], which assumes the liquid and gas phases are locally mixed. Relative to the sharp-interface capturing approaches, these methods provide a more computationally efficient means to model the spray. However, although such high fidelity modeling approaches can offer insight into the details of the spray formation process, their computational expense currently prohibits their application to engine simulations, where the evolution of the spray must ultimately be coupled with evaporation, fuel-air mixing, and combustion chemistry.

Due to the multi-phase, multi-physics, and multi-scale nature of the spray combustion process in direct injection engines, the most commonly employed spray modeling approach utilizes the Lagrangian-Eulerian framework $[14,15]$. In this method, the gas phase is resolved on the Eulerian grid while the liquid phase is modeled by tracking discrete parcels and their evolution using a Lagrangian formulation. Because the liquid phase is not directly resolved on the grid, there is a need to employ sub-models to represent the unresolved physics, such as primary and secondary break up, coalescence, evaporation, etc. 
However, the physical mechanisms governing atomization and spray formation and their characteristic length and time scales are still largely unknown due to the difficulty in directly observing these processes. Ideally, the spray breakup process would be quantified directly using imaging techniques. For high injection pressure sprays typical of CI engines, length and time scales characterizing the spray development are beyond the spatio-temporal resolution capabilities of current imaging systems [16-19]. Moreover, the proliferation of phase interfaces in such sprays makes them quite optically opaque, limiting the utility of many common optical diagnostics, especially in the spray formation region. Therefore, experimental approaches other than imaging are needed to study and characterize spray breakup for high injection pressure sprays under engine-relevant conditions.

In order to quantify details of the spray in the near-nozzle region where primary droplets are formed, alternative diagnostics to conventional imaging and droplet sizing techniques must be employed. X-ray radiography is an absorption-based technique [20,21], which can quantify the path-integrated liquid fuel mass distribution in a spray, commonly referred to as projected density. X-rays scatter quite weakly compared to visible light, and therefore can penetrate denser portions of the spray than what has been possible from conventional optical techniques. As a result, liquid mass distributions can be quantified throughout the spray, particularly in the near-nozzle region. Although $\mathrm{X}$-ray radiography cannot directly quantify spray structure, as it is a joint function of droplet size and number density, it does provide unique and insightful information in regions of the spray where primary breakup is expected to occur. Recent developments in X-ray diagnostics for sprays have demonstrated the capability of ultra-small angle $\mathrm{x}$-ray scattering (USAXS) techniques to make spatially-resolved, temporally-averaged interfacial surface area in high pressure diesel sprays [22]. These measurements can be combined with the x-ray radiography technique to quantify droplet size. The combination of these measurements affords a unique opportunity to use these newly quantified spray parameters, particularly in the near-nozzle region, to characterize the spray development process and assess existing spray modeling approaches of varying levels of fidelity and detail.

The above discussion clearly highlights the fact that joint experimental and modeling efforts can help improve current understanding of spray processes and how to best represent them in the context of engine simulations. To address this need, the Engine Combustion Network (ECN) was formed to formalize collaborations among national laboratories, academia, and industry [23]. Using classes of injectors with a set of nominally identical geometries (e.g. "Spray A," "Spray D," etc.), experimental and computational efforts have resulted in improved understanding of diesel and gasoline spray combustion. At the $5^{\text {th }} \mathrm{ECN}$ workshop, a dedicated session on primary atomization studied the spray formed from the ECN Spray D injector in subcritical conditions. This paper reports the major findings from different research groups which contributed to the session with simulations and experiments. X-ray experiments were conducted by Argonne National Laboratory, while simulations were presented by Georgia Institute of Technology, the Polytechnic University of Valencia (CMT), the University of Perugia, and Sandia National Laboratories. Various types of calculations, with increasing fidelity, ranging from Lagrangian, to Eulerian $\Sigma$-Y, VOF/LES and CLSVOF, are presented and compared against unique $\mathrm{x}$-ray measurements of distributions of liquid mass, phase interfacial area, and average droplet size. This type of quantitative data for a high-speed, optically-dense spray, and the detailed interpretation allowed by the high-fidelity simulations, along with the discussion for pathways to improve engineering-level spray models, are believed to be novel contributions of this work toward the general understanding of diesel fuel spray atomization.

Page 2 of 14
The main objectives of this joint experimental and computational effort were to: (1) unravel the primary breakup characteristics in terms of 2D distributions of mass, interfacial area and drop size in the dense near-nozzle region of a diesel injector; (2) compare different simulation approaches against these spray data; (3) leverage highfidelity simulation results to explain and support the experimental findings.

The paper is organized as follows. First, the x-ray based experimental measurement techniques are presented, followed by a brief presentation of each modeling approach. Novel aspects are discussed in detail, while aspects that have been already published are only referenced. Then, the results section presents comparisons of measured and predicted distributions of liquid fuel mass, interfacial area and droplet SMD, in the form of axial and radial profiles. The model predictions are used to interpret features in the experimentally observed spray structure, as well as to inform pathways to improve modeling approaches across the four methods presented in this work. The major conclusions and contributions of this work are then summarized in the final section.

\section{STANDARD COLD CONDITION FOR THE ECN SPRAY D INJECTOR}

The experimental and simulated condition selected to study the spray structure of non-vaporizing diesel sprays is detailed in Table 1. The ECN Spray D injector nozzle \#209133 is utilized in this work, which features a single-orifice diesel injector with a nominal diameter of $180 \mu \mathrm{m}$ and geometric K-factor of 1.5 , available to all participants of the ECN [23]. It should be noted that all experimental measurements were conducted during the steady portion of the spray event, when the injector needle is fully lifted and the injection velocity has reached a nominally constant value. Under these conditions, hydraulic characterization measurements $[24,25]$ indicate that the Spray D injector nozzle does not exhibit cavitation.

Table 1. Reference injection and ambient condition measured and modeled using the Engine Combustion Network Spray D injector [23]

\begin{tabular}{|c|c|}
\hline Fuel & n-dodecane \\
\hline Orifice Diameter (nominal) & $180 \mu \mathrm{m}$ \\
\hline K-factor (nominal) & 1.5 \\
\hline Injection Pressure & $150 \mathrm{MPa}$ \\
\hline Fuel Temperature & $298 \mathrm{~K}$ \\
\hline Ambient Temperature & $298 \mathrm{~K}$ \\
\hline Ambient Density & $22.8 \mathrm{~kg} / \mathrm{m}^{3}$ \\
\hline
\end{tabular}

\section{EXPERIMENTAL METHODS}

Details regarding the x-ray measurements performed on the Spray D injector at the Argonne Advanced Photon Source (APS) are provided in the following sections.

For both $\mathrm{x}$-ray radiography and ultra-small angle $\mathrm{x}$-ray scattering (USAXS) measurements, the Spray D injector was horizontally mounted in a pressure chamber fitted with a pair of $12 \times 30 \mathrm{~mm}$ x-ray transparent windows. The chamber was pressurized to the desired back pressure with $\mathrm{N}_{2}$, which was also used to maintain a continuous purge flow of approximately $1.5-4.0$ standard $\mathrm{L} \mathrm{min}^{-1}$ through the chamber to minimize droplet formation on the windows during data acquisition. 
A diesel common-rail injection system was used to pressurize ndodecane fuel to the desired rail pressure. The injector was fired at $3 \mathrm{~Hz}$ for a commanded injection duration of $2.0 \mathrm{~ms}$.

\section{X-ray Radiography}

Detailed descriptions of the time-resolved radiography measurements may be found in previous work [26, 27]. In brief, a monochromatic beam at $8 \mathrm{keV}$ energy passed through a set of curved mirrors, which focused the beam to a $5 \mu \mathrm{m} \times 6 \mu \mathrm{m}$ point. The incoming beam intensity, $I 0$, was measured using a diamond x-ray photodiode placed upstream of the pressure chamber. The transmitted beam intensity, $I$, downstream of the pressure chamber was measured with a PIN diode. As the $\mathrm{x}$-ray beam passed through the fuel spray, photons were absorbed primarily through the process of photoelectric absorption, attenuating the beam by an amount related to the amount of fuel in the beam path. From the change in beam intensity, the pathlength, $l$, of fuel in the beam path can be determined with the Beer-Lambert law,

$$
l=\frac{1}{\mu \rho_{\mathrm{f}}} \log \left[\frac{I_{0}}{I}\right]
$$

where $\rho_{f}$ and $\mu$ are the density and attenuation coefficient of the fuel, respectively. Between 16 and 32 spray events were averaged at each measurement point, and the x-ray beam was raster scanned in both the axial and transverse coordinates to create an ensemble-averaged map of the line-of-sight pathlength of fuel. While radiography data were acquired with a time resolution of $3.68 \mu \mathrm{s}$ after data processing, the data were averaged across the same time as the USAXS data acquisition for use in interpreting the USAXS data for droplet size.

\section{Ultra-Small Angle X-ray Scattering (USAXS)}

USAXS measurements were performed at the 9-ID beamline of the Advanced Photon Source (APS) at Argonne [28]. The x-ray beam was set to an energy of $21 \mathrm{keV}$ and a spot size of $50 \mu \mathrm{m} \times 500 \mu \mathrm{m}(\mathrm{V} \mathrm{x} \mathrm{H})$. The relative orientation of the beam with respect to the spray is illustrated in Figure 1. More information with respect to the USAXS technique may be found in previous literature [29-32], including its application to diesel fuel sprays [22]. Data acquisition was gated between $1.3-2.3 \mathrm{~ms}$ from the commanded start of injection; the gating ensured that the spray was in steady-state during measurements. A background signal was measured prior to each USAXS scan in order to eliminate the effect of residual spray droplets that settled within the measurement domain; in general, this background signal closely resembled a trace taken before any injection had taken place, indicating that few droplets were present in the chamber compared to those found in the spray during injection. The mean scattering intensity from the fuel droplets within the $50 \mu \mathrm{m} \times 500 \mu \mathrm{m}$ beam footprint was recorded at multiple transverse locations across the spray at a fixed scattering vector of $\mathrm{q}=3.0 \mathrm{e}-4 \AA^{-1}$. This transverse scan was repeated for multiple axial locations downstream from the injector tip. Using the Irena data analysis package in Igor [30], the resulting scattering intensity was converted to a projected surface area measurement, which quantifies the total interfacial surface area per beam area.

By combining the surface area measured with USAXS and the density measured with radiography, the Sauter mean diameter (SMD), or $d_{32}$, of the droplet size distribution can be determined. The transverse profiles from the USAXS and radiography measurements were each centered about their full width at half maximum in order to index the profiles onto the same coordinate system. Because the transverse location of the USAXS measurement is known at each axial distance,

Page 3 of 14 the corresponding radiography data at that location may be found. The USAXS measurement point is assumed to be in the center of the $50 \mu \mathrm{m}$ $\times 500 \mu \mathrm{m}$ window. All measured radiography points that fall within this window are averaged to arrive at one value of the pathlength, with interpolation and appropriate weighted averaging performed to accurately incorporate the edges of the window. The pathlength of fuel obtained from the radiography measurements provides the line-ofsight integrated volume of droplets. The USAXS measurements provide the line-of-sight integrated interfacial area per beam area. Thus, the two measurements can be combined per Equation 2 to arrive at a line-of-sight integrated SMD value at each measured axial location,

$$
d_{32}=6 \frac{V}{A}
$$

where $V$ and $A$ are the total volume and surface area of the group of particles within the measurement volume, respectively.

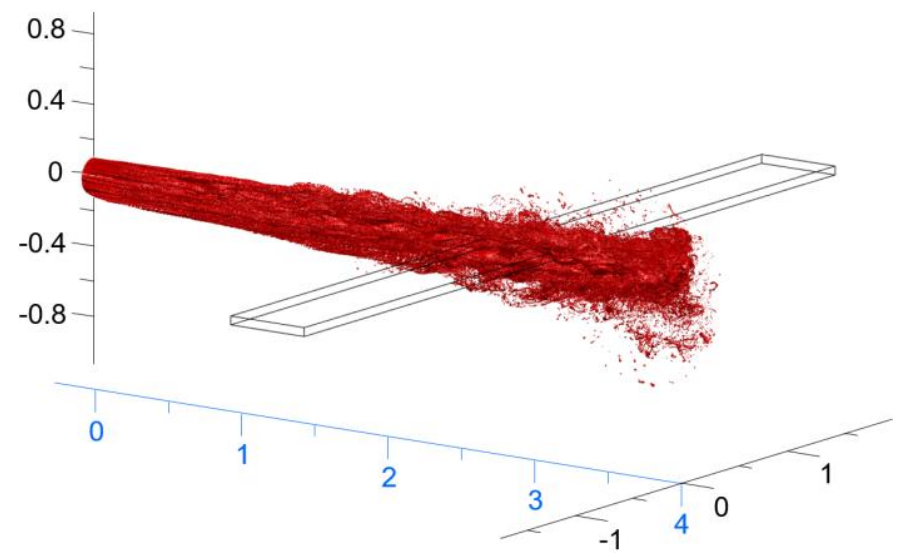

Figure 1. Schematic of the x-ray beam footprint for the USAXS measurement technique with respect to the orientation of the spray, where the distances along the axes are provided in terms of millimeters.

\section{NUMERICAL METHODS}

This section summarizes the four different numerical methods that are evaluated in this work to represent the fuel injection and spray formation processes. Sample visualizations of the predicted spray are shown in Figure 2 for the (a) Lagrangian-Eulerian, (b) Eulerian $\Sigma$-Y, (c) VOF-LES and (d) CLSVOF approaches. These snapshots highlight the level of detail that can be extracted with sufficient computational effort from each modeling approach. It should be noted that the spray visualizations from the four modeling approaches are not shown with the same scale. This section is organized in order of increasing modeling fidelity and computational effort, beginning with the Lagrangian-Eulerian approach and finishing with the CLSVOF method. Salient differences among the numerical frameworks, such as internal nozzle flow modeling, treatment of the liquid-gas interface, and turbulence modeling approaches, are also noted. 


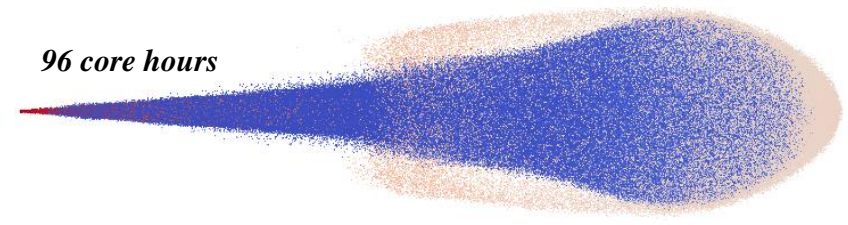

(a)

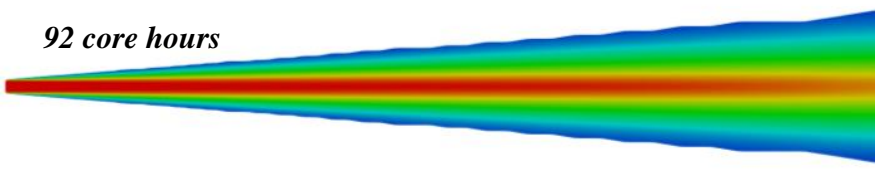

(b)

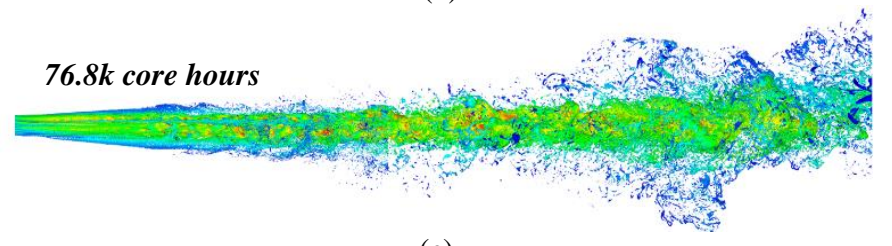

(c)

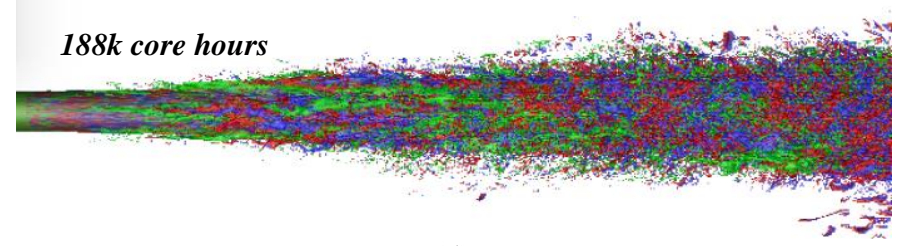

(d)

Figure 2. Visualizations of the predicted spray from the (a) LagrangianEulerian, (b) Eulerian $\Sigma$-Y, (c) Volume of Fluid - Large Eddy Simulation (VOF-LES) and (d) Coupled Level Set - Volume of Fluid (CLSVOF) modeling approaches. The total CPU time is overlaid for each modeling approach to highlight the trade-off between predicted detail and computational effort.

\section{Georgia Institute of Technology, USA (Lagrangian)}

The Lagrangian-Eulerian model formulation employed to represent the injection of an n-dodecane spray into a constant volume chamber has been extensively assessed and validated by the authors from Georgia Institute of Technology against a range of spray measurements, such as liquid penetration, projected density, and USAXS-derived SMD distributions [33-34]. Details of the spray model set-up employed in CONVERGE have been discussed in previous publications, but the salient features pertinent to the spray atomization model are summarized here. Using the "blob" injection model [15], injection of 1 million computational parcels was determined to be sufficient to statistically represent the dense spray [33]. Instead of modeling the internal nozzle flow development within the injector, the initial velocity of the injected parcels is defined at the nozzle exit boundary using the measured fuel mass flow rate and nozzle discharge coefficient [25]. Liquid mass is injected within a circle instead of at a point source to yield better mass distribution in the near-nozzle region [35], where the radius of the circle is equated with the nozzle radius, as measured by the detailed x-ray tomography scan of the Spray D \#209133 injector [36]. Primary and secondary spray breakup were modeled with the KH-RT model [37], while the influence of droplet collisions and coalescence was neglected. Careful calibration of the model constants characterizing the spray breakup time and equilibrium droplet size against USAXS-derived SMD measurements for the ECN Spray A injector over a range of injection and ambient densities yielded good agreement between the measured and predicted centerline SMD profiles [34]. The same constants are employed for the Spray D injector modeled in this study. Other implemented sub-models Page 4 of 14 include the Reynolds-Averaged Navier-Stokes (RANS) standard k- $\varepsilon$ turbulence model [38] with a turbulent round-jet correction [39] to represent the flow in the gaseous ambient environment.

The computational grid employed for the Lagrangian-Eulerian spray predictions is shown in Figure 3(a). To obtain sufficient grid resolution in the near-nozzle region while maintaining a reasonable total cell count, fixed embedding and adaptive mesh refinement were employed to yield a minimum grid resolution of $125 \mu \mathrm{m}$. The spray features of interest were extracted by mapping the Lagrangian parcel information to a post-processing grid with a spatial resolution of $100 \mu \mathrm{m}$. This information was then time-averaged during the steady portion of injection from $0.5-1.0 \mathrm{~ms}$, with a sampling frequency of $0.05 \mathrm{~ms}$.

\section{Universitat Politecnica de Valencia, Spain ( $\Sigma-Y)$}

To perform the simulations, an Eulerian homogeneous unsteady multiphase model has been implemented in OpenFOAM Foam-extend 3.2, similar to the one developed by Vallet et al. [11]. The three main differences are: the pressure equation corrected by García-Oliver et al. [12] is used instead of the equation of state of Vallet et al.; the new model is compressible, so the energy equation is needed (in this case it is written in terms of static enthalpy) [13]; and liquid density and compressibility are computed through correlations to experimental data $[13,40]$.

Even though the model has been thoroughly described in the literature and previous publications $[12,13]$, a brief summary of the equations and sub-models is given. As previously mentioned, the liquid spreading into the ambient gas is modeled with a turbulent diffusion flux given by Fick's law. A RANS 2-equation viscosity model is employed, namely the standard k- $\varepsilon$ model, to estimate the turbulent viscosity $\mu_{\mathrm{t}}$ and model the gas-phase flow field. Once the liquid mass fraction is obtained, the density of the liquid-gas mixture, $\rho$, is calculated through:

$$
\rho=\frac{1}{\frac{Y}{\rho_{l}}+\frac{1-Y}{\rho_{g}}}
$$

where $Y$ is the liquid mass fraction, $\rho_{l}$ and $\rho_{g}$ are the liquid and gasphase densities, respectively. In combination with the liquid dispersion transport equation, the small-scale atomization is modeled by solving another transport equation for the evolution of the density of interphase surface area, as originally proposed by Vallet et al. [11], and given by Equation (4).

$$
\frac{\partial(\rho \Sigma)}{\partial t}+\nabla \cdot(\rho \vec{U} \Sigma)-\nabla \cdot\left(D_{\Sigma} \nabla \Sigma\right)=(A+a) \Sigma-V_{s} \Sigma^{2}
$$

Coefficients $A$ and $a$ account for interphase surface generation due to atomization, while coefficient $V_{s}$ models the destruction of surface density mainly due to coalescence. Once again, the reader is referred to the literature [12] for a more detailed description of these coefficients.

Since a RANS approach is used for the turbulence, and the Spray D geometry is almost axisymmetric, a 2D axisymmetric domain is selected in order to save computational time. The computational mesh is shown in Figure 3(b), and includes information for a portion of the injector, based on nominal geometry specifications [23], as well as the discharge chamber. For the discharge chamber, dimensions of $12 \mathrm{~mm}$ x $6 \mathrm{~mm}$ are selected to model a free fuel jet where wall effects are insignificant within the timescales of interest. After performing a grid 
sensitivity analysis, the mesh cell count is 54423, with a minimum cell size of $1 \mu \mathrm{m}$ located at the orifice outlet, and a maximum cell size of $450 \mu \mathrm{m}$.

Post-processing of the spray features of interest are detailed here. The predicted distributions of interfacial surface area are determined through instantaneous spatial distributions of $\Sigma$. SMD is directly computed, as described in the work of Vallet et al. [11]. For projected quantities, axisymmetry is assumed to reconstruct the full $3 \mathrm{D}$ distribution, and then line-of-sight integrations are conducted to determine the $2 \mathrm{D}$ distributions.

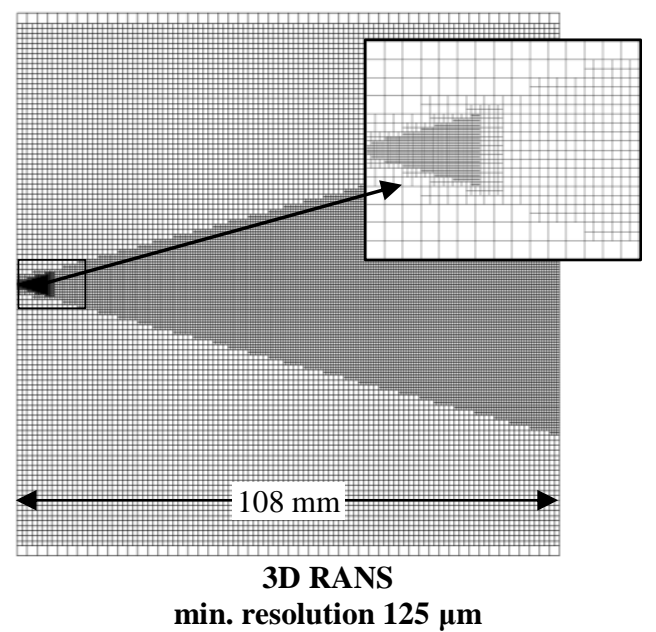

(a)

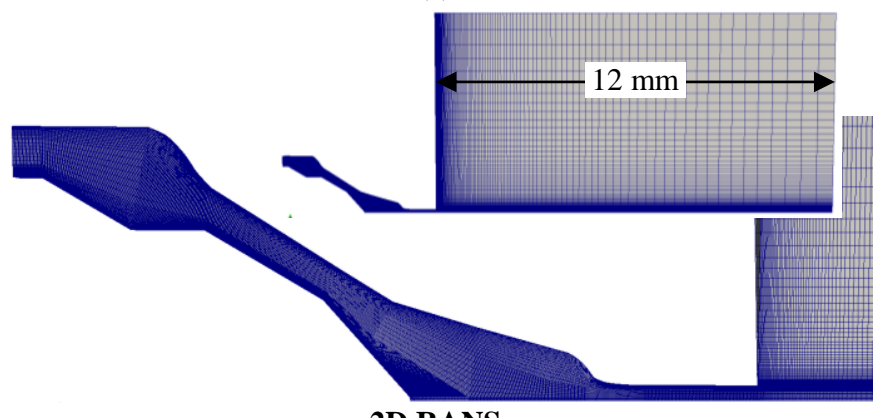

2D RANS

min. resolution $1 \mu \mathrm{m}$

(b)

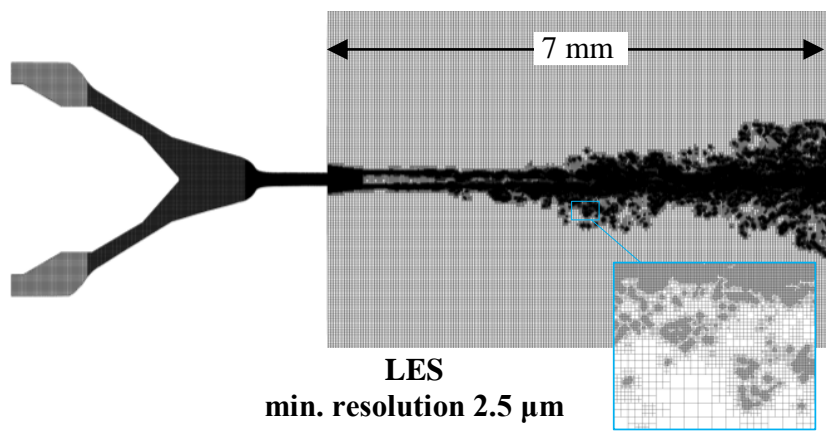

(c)

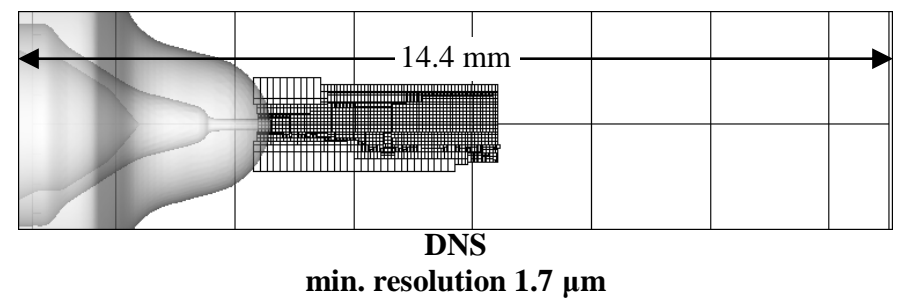

(d)

Figure 3. Computational meshes employed for the (a) Lagrangian, (b) Eulerian $\Sigma$-Y, (c) VOF-LES and (d) CLSVOF modeling approaches. Information is also provided regarding the employed turbulence model and minimum cell size.

\section{Università degli Studi di Perugia, Italy (VOF-LES)}

An Eulerian method, which enforces and maintains a sharp interface between the two phases, is employed to model the fuel injection and spray evolution. With this approach, liquid and gas phases are regarded as immiscible. The formulation is based on the volume-of-fluid (VOF) methodology, well established in the literature [10]. The mathematical model is implemented in the commercial code CONVERGE, which is based on a convenient cut-cell Cartesian method for generating the grid at run time [41]. In addition to mass and momentum, the VOF solution method directly transports by convection the volume fraction of one of the two phases, without any diffusion or source term. This constraint allows the method to maintain sharp phase interfaces. In this work, the energy equation is not solved and only two incompressible fluids, fuel and air, are considered. The effect of surface tension is included in the momentum equation with the continuum surface force model [42]. The phase interface is reconstructed with a Piecewise-Linear Interface Calculation (PLIC) method. Specifically, the procedure goes through successive steps. First, the surface normal is estimated based on the gradient of the volume fraction. Second, a planar approximation is made locally in each computational cell and fitted to satisfy mass conservation. Third, the interface is advected, via a 3D split advection scheme $[10,43]$. Fluid densities and viscosities are constant in the pure phases, while across the interface a linear average based on the local volume fraction is adopted. Surface tension is also assumed to be constant.

Despite the fine spatial resolution of the employed computational mesh, sub-grid stresses in the momentum equation are not considered negligible. To account for non-resolved sub-grid-scale (sgs) fluctuations in the pure phases the Dynamic Structure LES turbulence model is included [44]. No attempts have been made though to include sgs effects on the phase interface, due to lack of established models. Along the same line of argument, wall functions are adopted, as the very high Reynolds number rules out any possibility of directly resolving the boundary layer on the orifice walls. This modeling approach will be referred to as VOF-LES, as also found in the literature [45]. The numerical solution of the conservation equations is based on the PISO algorithm for momentum-continuity coupling. Second order central-differencing with flux-limiter is used for spatial discretization of each equation, with an Euler scheme for the time discretization. This numerical framework has been presented and validated in previous works [46-48].

The three-dimensional computational domain is shown in Figure 3(c). The detailed internal nozzle boundary surface was obtained from x-ray tomography scans of the Spray D \#209134 injector [49], which has a nominally identical geometry as the Spray D \#209133 injector employed in the x-ray experiments. A minimum grid resolution of $2.5 \mu \mathrm{m}$ is obtained through the application of AMR based on volume 
fraction and velocity gradients, which extends far downstream. This approach allows the number of cells to be limited to 60 million, while capturing the phase interface with sufficient accuracy. In the current simulation, the time-step is below $10^{-9} \mathrm{~s}$, controlled by a convective CFL of 0.2. Fluctuations of the resolved velocity field at the inlet boundary, located upstream of the needle, are neglected, considering that most of the turbulence is generated through the internal passages. Simulations are transient with fixed needle at high lift, and are run until main flow variables stabilize.

Post-processing of the results obtained with the interface-capturing method deserves a brief discussion. The objective is the identification of each disconnected liquid structure and the calculation of its volume and surface area. Because the simulation adopts an AMR based mesh, the first step is the resampling of the region of interest with a uniform grid having the size of the smallest cells. This does not involve interpolation because the grid is perfectly Cartesian. Second, a binarization of the volume fraction field is performed, with a threshold of 0.5 . Third, a connectivity algorithm looks for detached structures and returns the number of cells belonging to each structure, from which the volume is obtained. Connectivity is based on all 26 neighboring nodes. Lastly, an iso-surface is constructed based on this data, and the surface area of each isolated liquid blob is computed. The evaluation of an equivalent SMD is conducted on specific sub-regions of interest (boxes, rings, line-of-sight prisms, etc.) and the value is computed using Equation (2).

\section{Sandia National Laboratories, USA (CLSVOF)}

The rapidly evolving, topologically convoluted surface that separates the liquid fuel from the gas is resolved with a time-accurate frontcapturing technique. The numerical methodology employed in this work assumes that the liquid surface is always well defined and that it separates two immiscible fluids. The CLSVOF code is a multi-phase compressible solver for the Navier-Stokes equations, which are solved with the mass-, momentum-, and energy-conserving advection algorithm described by Jemison et al. [9]. Because no model is imposed to represent turbulence in the CLSVOF framework, fine grid resolution is employed to directly resolve the fluid motion, akin to DNS. The solution is advanced in time by a semi-implicit pressure update scheme that asymptotically preserves the standard incompressible pressure projection in the limit of infinite sound speed; see Kwatra et al. [50] for its original single-phase formulation. This hybrid approach makes interface capturing methods applicable to compressible flows (the user can actually choose which fluid behaves as compressible), while using time steps that can be larger compared to typical explicit methods.

The computational setting follows the one adopted for a recent study of n-dodecane spray atomization [51], including the fitted equation of state for $\mathrm{n}$-dodecane described therein. Equations are discretized on regular Cartesian computational cells. The level-set function is maintained at each time step as the signed distance to the reconstructed liquid-gas surface. The curvature of the liquid surface is evaluated from the level set function by using the method of heights [52].

The computational mesh was constructed in a similar manner as the one employed in the VOF-LES approach, where the injector boundary surface was obtained from detailed x-ray tomography scans of the ECN Spray D \#134 injector [49]. The computational domain shown in Figure 3(d) illustrates the edges of the first three levels of Cartesian blocks within the domain for the fuel nozzle and ambient environment. Since a Cartesian mesh cannot be body-fitted to complex boundary geometries, the injector's walls are represented by the embedded boundary method described in Arienti and Sussman [53]. Dynamic adaptive mesh refinement (AMR) is used to concentrate the computational resources at interfaces, and relatively good scaling has been tested with up to 10,000 processes. The computational mesh is a box of length $1.44 \mathrm{~cm}$ in the axial direction and $0.36 \times 0.36 \mathrm{~cm}$ in the transverse direction; on this base layer, additional levels of refinement are added following the Berger-Colella algorithm.

In the post-processing stage, the zero iso-surface of the liquid-gas level set (the liquid surface) is transformed into a tessellation made of triangular or quadrilateral faces. Contrary to diffuse-interface methods, the surface is calculated unambiguously, as the sharp separation between the values zero and one of liquid volume fraction. A recursive procedure then separates from each other structures that do not have nodes in common, generating polyhedra that correspond each to a well-defined "blob" shape. The number of faces of each blob may vary from several thousands to a few tens. Choosing a sufficiently high cutoff number, say 48 faces, ensures that under-resolved structures of the liquid surface are discarded. In this approach, the SMD can be calculated using Equation (2) for the detached drops or ligaments, without making assumptions about the shape of the liquid structures. The evaluation of the liquid surface density, indicated by the symbol $\Sigma$, is based on the same tessellation of the liquid surface. For simplicity, the tangential average is considered by taking an annular probe of volume $V_{q}$ located at the distance $r_{q}$ from the axis. At any point of coordinates $r_{q}$ and $x_{q}$, one can calculate $A_{q}$ as the sum of the areas of the faces contained in the probe region. As a result, $\Sigma$ can then be calculated using Equation (5):

$$
\Sigma=A_{q} / V_{q}
$$

It is noted that, because of the short observation time in this computationally expensive simulation, a sufficiently large statistical sample is found by looking at all the ligaments and drops that fall in a relatively large spray region (of the order of ten orifice diameters).

\section{RESULTS AND DISCUSSION}

In order to evaluate the atomization processes and resultant spray structures predicted by the four different modeling approaches, measured and predicted spray quantities are compared. This section is organized by first evaluating spray parameters describing the liquid fuel mass distributions, namely the axial transverse integrated mass (TIM) and radial projected density profiles. Predicted and measured projected surface area distributions are then compared. The final section evaluates the details of the measured and predicted spray morphology through comparison of the SMD distributions throughout the spray.

The experimentally measured and predicted TIM are compared in Figure 4. TIM quantifies the total amount of liquid-phase fuel mass present per unit length in the axial direction. Good agreement can be seen between the experimental measurements and model predictions. As shown previously by Kastengren and co-workers [54], using the continuity equation it can be shown that TIM is inversely proportional to the mass-weighted transverse-averaged axial velocity, thereby indicating that all models predict similar axial velocities of the liquidphase, particularly within the first $4 \mathrm{~mm}$ of the spray. 


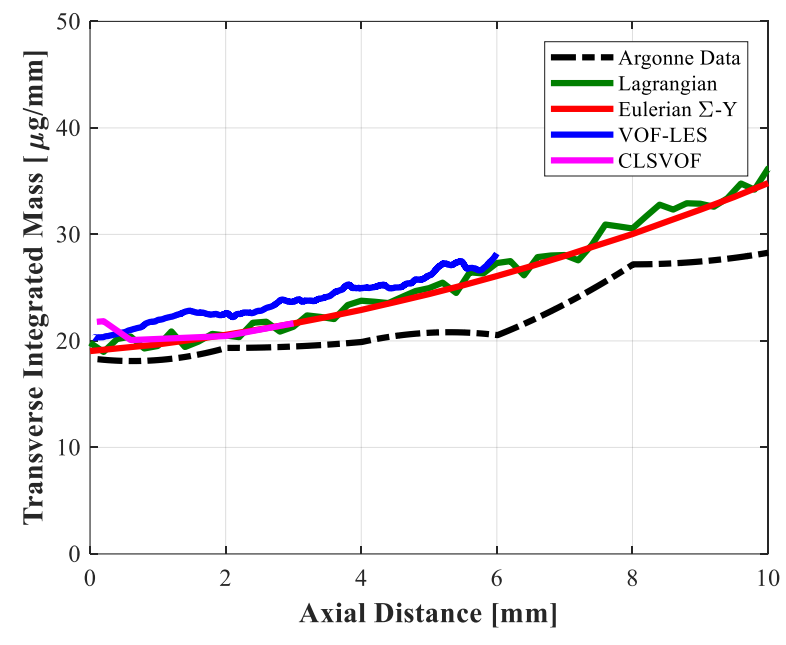

Figure 4. Experimentally measured and predicted transverse integrated mass (TIM) under the standard cold condition for the ECN Spray D injector.

Experimentally measured projected density profiles within the first $4 \mathrm{~mm}$ from the nozzle exit are compared with model predictions, as shown in Figure 5 for axial positions of (a) $0.1 \mathrm{~mm}$, (b) $2.0 \mathrm{~mm}$, and (c) $4.0 \mathrm{~mm}$. In general, the higher-fidelity modeling approaches $(\Sigma-Y$, VOF-LES, and CLSVOF) are able to capture both the peak projected density at the spray centerline, as well as width of the spray within the near-nozzle region. It is worth noting that the measured projected density profile at an axial position of $2.0 \mathrm{~mm}$ in Figure 5(b) is particularly asymmetric, and exhibits two peaks in the transverse distribution. The ability of the VOF-LES approach to replicate this feature in the projected density distribution may be attributed to the detailed nozzle geometry [49] that was employed to model the internal nozzle flow development within the injector. Although the CLSVOF approach also employed the same detailed x-ray scan in constructing the internal injector boundary surfaces, a relatively symmetric projected density distribution is predicted instead. The observed differences in the predicted profiles is likely due to the differences in the processing routines. For example, for the VOF-LES predictions, a single viewing angle was utilized when calculating the projected density distributions. In contrast, the projected density profiles from the CLSVOF simulations were processed by considering several viewing angles in order to achieve a sufficient number of samples. This processing routine would result in a relatively symmetric profile, similar to the predictions from the Lagrangian and two-dimensional Eulerian $\Sigma$-Y approaches. Future work should consider the influence of viewing angle on the ability to capture experimentally observed asymmetries in the projected density profiles, and explore the correlation of asymmetry in the $\mathrm{x}$-ray radiography data with geometric features from the x-ray tomography scans.

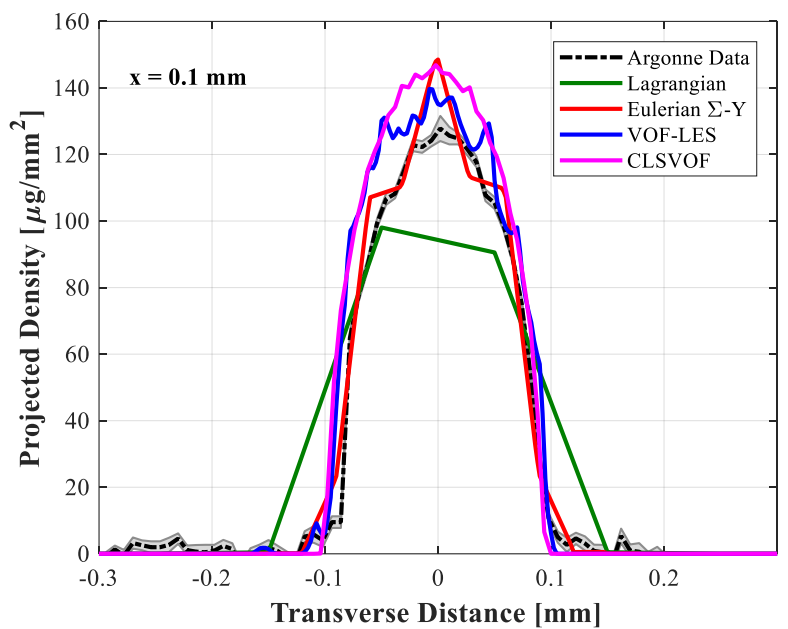

(a)

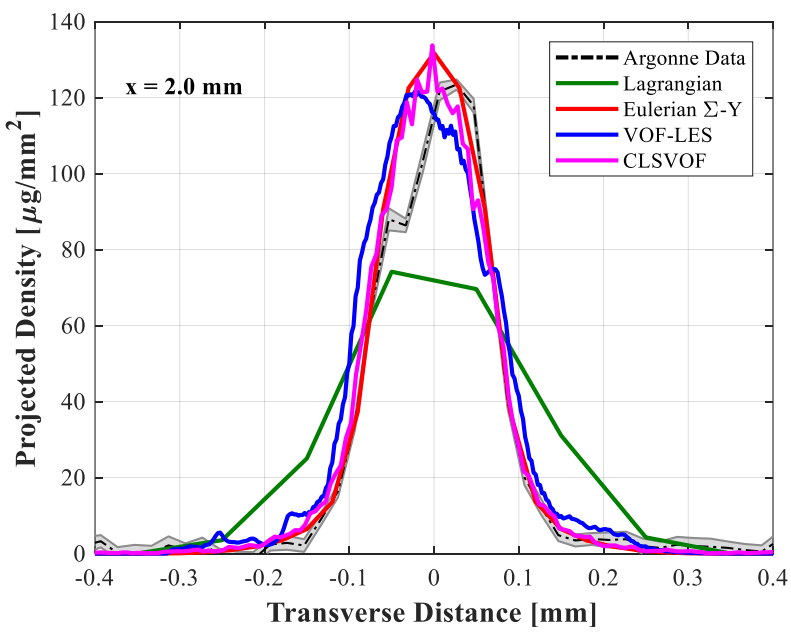

(b)

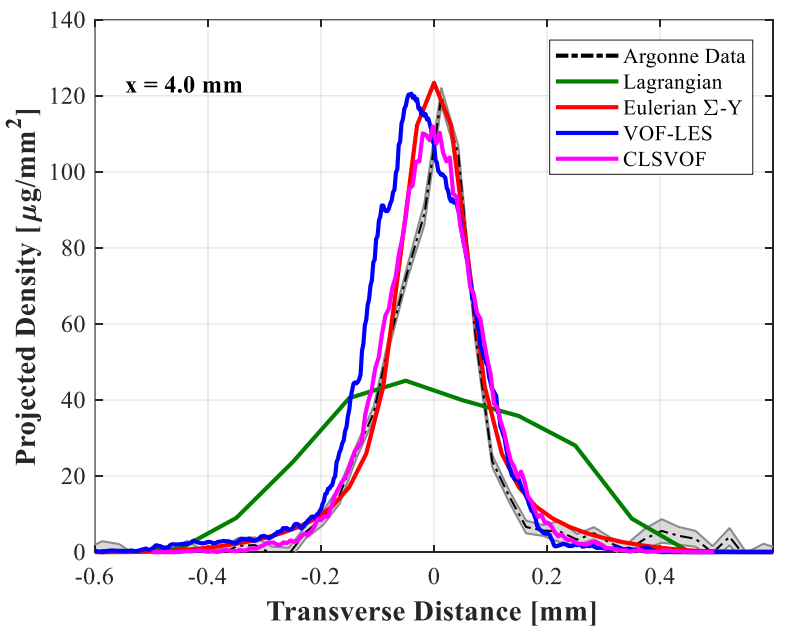

(c)

Figure 5. Experimentally measured and predicted projected density distributions at distances of (a) $0.1 \mathrm{~mm}$, (b) $2.0 \mathrm{~mm}$, and (c) $4.0 \mathrm{~mm}$ from the injector nozzle exit. Standard deviation of the data is represented by the gray shaded region.

Page 7 of 14 
Although the Lagrangian-Eulerian approach is able to predict a similar TIM as the other modeling approaches, the peak values of the projected density profiles are underpredicted and the dispersion of the spray is overpredicted. The underlying sources for this discrepancy have been discussed at length in the literature $[47,55]$, and in general have been attributed to the challenges of sampling the Lagrangian computational parcels at a consistent spatial resolution as the experimental measurements, as well as to the treatment of the liquid-gas coupling in the modeling framework. Even with improved liquid-gas coupling methods and the use of fine spatial discretization in the near-nozzle region [35], the Lagrangian-Eulerian method overpredicts dispersion of the spray. Improvements to the turbulent-dispersion sub-model formulation, which represents the influence of gas-phase velocity fluctuations on the trajectory of Lagrangian computational parcels, could help improve predictions of the liquid mass distribution for the Lagrangian-Eulerian formulation. The range of modeling approaches, from engineering (Lagrangian) to scientific (CLSVOF) level calculation, applied at this well-characterized condition creates a unique opportunity to improve turbulent-dispersion sub-model formulations. Because all three higher fidelity approaches are able to capture the near-nozzle liquid mass distribution, the gas-phase velocity fluctuations and induced liquid-phase motion can be sampled to assess and improve turbulent-dispersion model formulation. Extension of this work to a range of injection and ambient conditions would create a pathway to improve existing turbulent-dispersion sub-models employed in engineering-level calculations.

The experimentally measured projected surface area by the USAXS technique at a fixed scattering vector, $q$, is shown for three different axial positions in Figure 6(a). These data can be compared to a reference projected surface area for a cylinder with equal diameter as the Spray D \#133 injector nozzle $(186 \mu \mathrm{m})$, centered within the footprint of the USAXS beam $(50 \mu \mathrm{m} \times 500 \mu \mathrm{m})$. This reference value of $2.03 \mathrm{~mm}^{2} / \mathrm{mm}^{2}$ quantifies the projected surface area for an undisturbed liquid column, and provides an estimate for the minimum projected surface area expected in the central region of the spray. Measured projected surface areas greater than this reference value along the spray centerline suggest that the probed measurement volume likely contains surface area information corresponding to detached liquid structures and droplets. At $2.0 \mathrm{~mm}$ from the nozzle exit, a local minimum in the projected surface area is observed at the spray centerline that is greater than this reference value. It is interesting to note that local minima in the transverse specific area profiles remain until axial distances greater than $6.0 \mathrm{~mm}$ from the injector nozzle exit. This pattern is distinct from the projected density distribution shown in Figure 5, where the peak in the mass distribution is consistently observed in the center of the spray. However, at further distances downstream of the nozzle, the characteristic shape of the projected surface area profile changes, as the peak surface area is observed to be co-located with the peak projected density at the spray centerline.

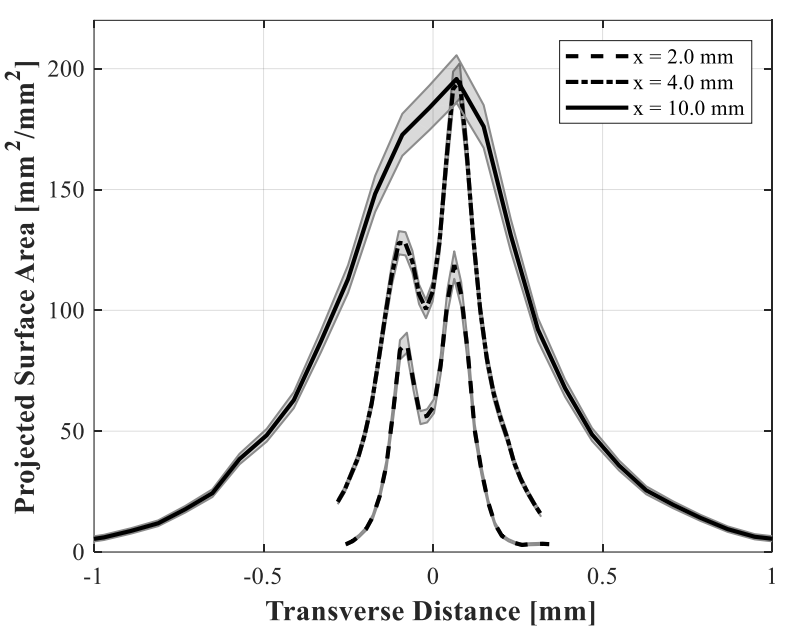

(a)

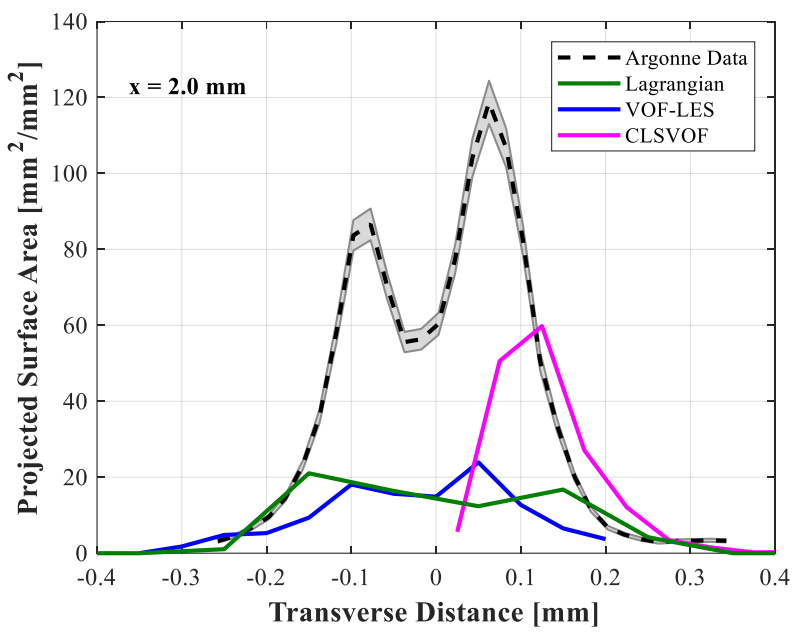

(b)

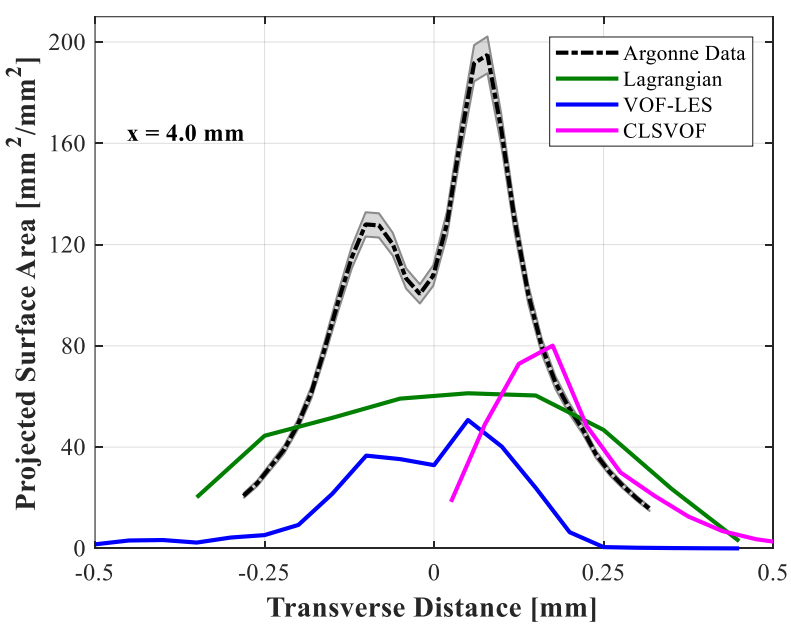

(c)

Figure 6. Transverse projected surface area profiles as (a) measured by the USAXS measurement technique at various axial distances, $\mathrm{x}$, from the injector nozzle exit. Predicted projected surface area distributions are shown at axial distances of (b) $2.0 \mathrm{~mm}$ and (c) $4.0 \mathrm{~mm}$ from the injector nozzle exit. Standard deviation of the data is represented by the gray shaded region.

Page 8 of 14 
To evaluate the reason behind this experimentally observed trend in further detail, the structure of the spray as predicted by the different modeling approaches is examined. The predicted transverse projected surface area profiles at axial positions of $2.0 \mathrm{~mm}$ and $4.0 \mathrm{~mm}$ from the nozzle exit are shown in Figure 6(b) and (c), respectively. At both axial locations, all models predict less surface area than is suggested by the experimental data at the spray centerline. Along the spray periphery, the CLSVOF approach captures the experimentally observed projected surface area profile. The VOF-LES approach consistently predicts less total surface area in comparison to the CLSVOF predictions, which can be attributed to the relatively larger resolution employed in the simulation $(2.5 \mu \mathrm{m}$ vs $1.7 \mu \mathrm{m})$. However, the locations of the peaks in the projected surface area profiles are faithfully reproduced at both axial positions. The Lagrangian spray model predicts similar levels of projected surface area as the VOF-LES approach in the central regions of the spray, and similar projected surface area levels as the CLSVOF along the spray periphery. Although the modeling approaches predict different magnitudes for the projected surface area at an axial position of $2.0 \mathrm{~mm}$ from the nozzle exit, all models predict a local minimum in the projected surface area near the spray centerline. These results indicate that although different degrees of atomization are predicted, similar spray structures are suggested by the models. However, further downstream at an axial position of $4.0 \mathrm{~mm}$, the Lagrangian spray model does not predict the bimodal projected area distribution observed in the experimental USAXS data and predicted by the other modeling approaches. Further calibration of the spray model constants to delay the spray breakup process would likely improve the predicted spray structure.

To evaluate the physical meaning of the predicted surface area profiles, the liquid structures predicted by the CLSVOF approach are shown in Figure 7(a). From the visualization, it is clear that the injected column of fuel is largely intact within the first $1 \mathrm{~mm}$ section from the nozzle, as denoted in cyan in the visualization. Within $2 \mathrm{~mm}$ from the nozzle, as denoted in green, the periphery of the spray is broken up into ligaments and droplets. As highlighted in Figure 7(b), the central region of the spray is devoid of droplets and detached structures due to the presence of a liquid core. It can be seen in Figure 6(b) that this region coincides with the local minimum in projected surface area predicted by the CLSVOF model, whereas regions comprised of droplets along the periphery result in local peaks in the projected surface area. This interpretation of the measured projected surface area distribution from the USAXS technique is consistent among all of the model predictions.

In short, the local minima near the spray centerline are due to the presence of larger liquid structures near the spray axis. When taking into account that the measured surface area data are a projection of the surface area distribution in the spray, the source of the bimodal pattern seen in the data can be easily understood, as schematically represented in Figure 7(d). In central regions of the spray, the projected surface area profile may be related to the degree of atomization of the liquid jet, where local minima may indicate regions of poorly atomized spray. This conclusion highlights the utility of the USAXS measurement to not only quantify information about the local surface area distribution throughout the spray, but also to qualitatively describe the structure of the spray.

Page 9 of 14

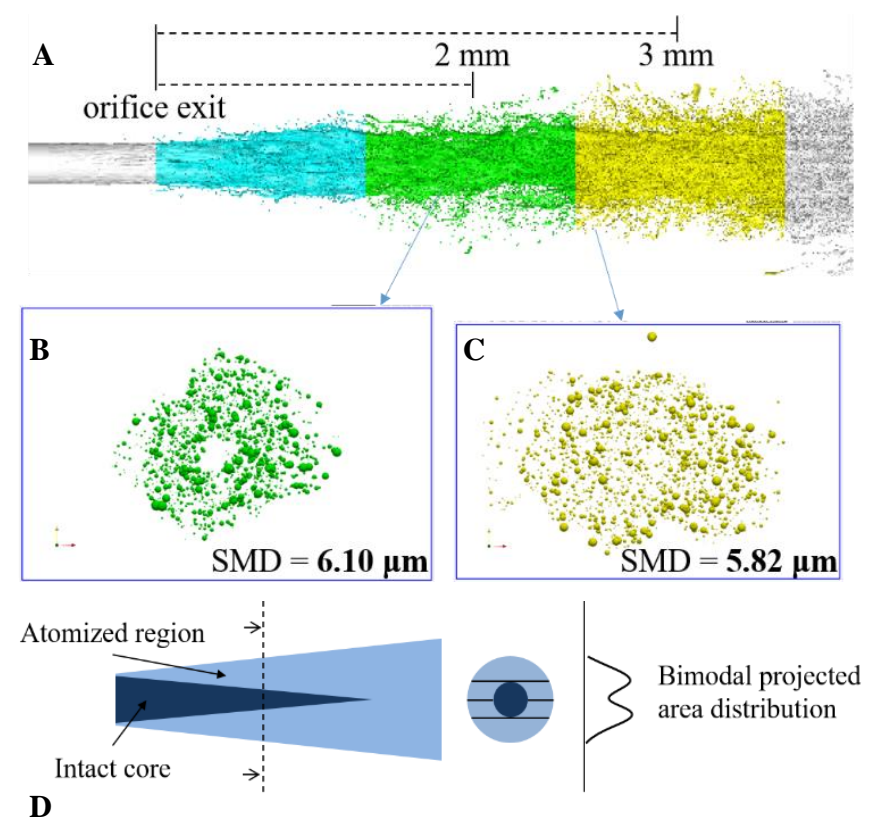

Figure 7. (a) Visualization of the CLSVOF spray prediction, and summary of droplet statistics within $1.0 \mathrm{~mm}$ wide bins. (b) At an axial position of $2.0 \mathrm{~mm}$, the center of the jet is occupied by the liquid core, and no droplets can be observed. Further from the orifice, droplets begin to form near the jet center, as can be seen in (c) at an axial position of $3.0 \mathrm{~mm}$ from the nozzle exit. (d) A schematic representation of the predicted spray highlights the reason for the observed projected surface area transverse profile.

As previously noted, the four different modeling approaches indicate different degrees of atomization within the first $2 \mathrm{~mm}$ from the injector nozzle exit. To further explore the differences in the resultant spray structure following atomization, the measured and predicted SMD profiles are compared. First, it is important to note that SMD information can only be extracted from the magnitude of the USAXS scattering signal when certain criteria are met, namely that droplets and other liquid structures are randomly oriented and that the scattering is axisymmetric [22]. Additionally, because the SMD distributions characterize the volume to surface area ratio across the width of the spray, it is possible that the SMD values average information from both well atomized and poorly atomized fuel, particularly in central regions of the spray near the injector nozzle exit. Because it is not possible to directly validate the occurrence of these conditions within the nearnozzle region, the measured SMD values in the near-nozzle region are interpreted with these factors in mind.

The measured transverse SMD distributions are shown in Figure 8(a) for axial positions of $2.0-14.0 \mathrm{~mm}$ from the injector nozzle exit. The peak SMD values in the center of the spray are used to define the axial SMD distribution along the spray centerline. For each transverse SMD distribution, a spatially-averaged SMD is also determined and compared with the centerline SMD, as shown in Figure 8(b). Comparison of the centerline and spatially-averaged SMD quantities allows for the spatial variation in SMD across the width of the spray to be evaluated. The USAXS measurements suggest a rapid decrease in the characteristic size of the spray within the first $2 \mathrm{~mm}$ from the nozzle exit, from a diameter of the injector nozzle $(186 \mu \mathrm{m})$ to approximately $15 \mu \mathrm{m}$. The SMD of the droplet size distribution continues to decrease in size until it reaches an approximately steady value of $2 \mu \mathrm{m}$ at distances of $10 \mathrm{~mm}$ and greater from the injector nozzle exit. At these downstream locations the centerline SMD is seen to converge with the spatially-averaged SMD values, indicating minimal spatial variation in SMD across the width of the spray. This trend is confirmed in the transverse SMD distribution shown in Figure 8(a), where the SMD 
distributions at axial positions of $10.0 \mathrm{~mm}$ and $14.0 \mathrm{~mm}$ are relatively constant across the width of the spray. The stable SMD value achieved in downstream portions of the spray are consistent with the SMD values reported along the periphery of the spray within the near-nozzle region $(1.5-2.0 \mu \mathrm{m})$. These findings suggest that no matter where ligaments start forming and detaching from the core, the resultant droplets have an almost uniform value.

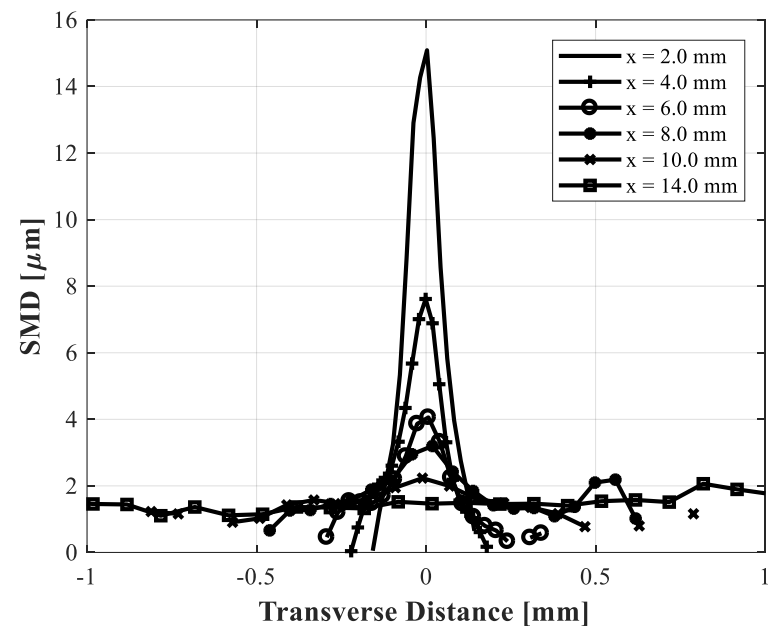

(a)

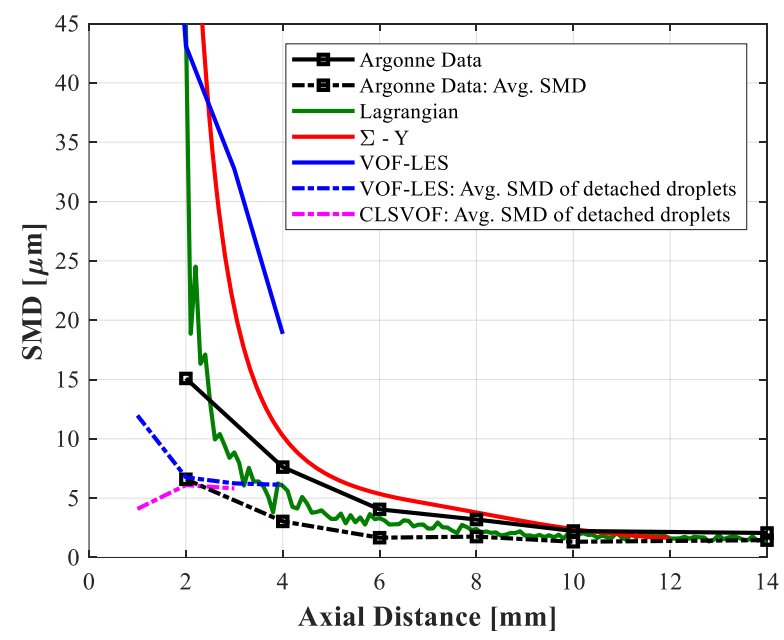

(b)

Figure 8. The experimentally determined transverse SMD profiles shown in (a) are used to determine the centerline SMD and spatially averaged SMD, as shown in (b) using solid and dashed lines, respectively, and compared with model predictions.

As shown in Figure 8(b), some differences are observed between the measured and predicted SMD profiles, particularly within the nearnozzle region. The measured SMD from the USAXS techniques is determined from the joint $\mathrm{x}$-ray radiography and scattering signal measurements, which quantify the total mass and surface area of all liquid structures within the respective measurement volumes. However, the post-processing routines for the different modeling approaches do not necessarily allow for as consistent of an evaluation of the spray as the experiments. For example, in the CLSVOF results, only detached droplets are considered in the calculation of SMD, therefore excluding the intact liquid core and attached ligaments from the calculation. As a result, within the first $3 \mathrm{~mm}$ of the near-nozzle region, the CLSVOF approach indicates much smaller SMD values than is indicated by the centerline SMD measurements. This may Page 10 of 14 suggest that the SMD measurements in the near-nozzle region may contain information for both atomized droplets and the intact liquid core within the measurement volumes, which would serve to increase the indicated SMD. This interpretation is strengthened through separately analyzing the SMD of the detached droplets predicted by the VOF-LES simulation. In comparison to the centerline SMD, the predicted SMD of the detached droplets are indeed much smaller in the near-nozzle region, and are only marginally larger than the CLSVOF predictions. To enable an improved assessment of the resolved droplet sizes by the CLSVOF and VOF-LES approaches, the predicted SMD of the droplet size distribution are compared with the spatiallyaveraged SMD derived from the measured transverse SMD profile. In the near-nozzle region, excellent agreement is obtained between the VOF results and the spatially-averaged SMD from the USAXS data.

Even though the post-processing routines for the other modeling approaches consider contributions from both the intact core and formed droplets on the SMD calculation, differences are observed among the predicted SMD profiles. Although the Lagrangian and VOF-LES modeling approaches predict similar SMD at $2.0 \mathrm{~mm}$ along the spray centerline, the VOF-LES model predicts a much slower atomization process as indicated by the larger SMD values relative to the Lagrangian model predictions and the experimental data. This may be due in part to the current resolution capabilities for the VOF model, where the minimum resolvable droplet size is approximately 5-6 $\mu \mathrm{m}$. Applying the VOF-LES approach with a finer grid resolution (less than $2 \mu \mathrm{m}$ ) would help detect the formation of smaller droplets, and likely yield improved agreement with the measured SMD profile. Downstream of the near-nozzle region, both the Eulerian $\Sigma-Y$ and Lagrangian modeling approaches match well the experimentally measured SMD and rate of droplet size decrease. In spite of the calibration that is required for both the Eulerian $\Sigma$-Y and Lagrangian modeling approaches to achieve good agreement with the experimental data, this comparison highlights the utility of detailed x-ray measurements and their ability to inform improvements to engineering-level models.

In particular, the ability of a Lagrangian spray simulation to capture the experimentally measured centerline SMD distributions relies heavily on the employed spray breakup sub-model. In previous studies conducted by Magnotti and Genzale [34], careful calibration of the $\mathrm{KH}-\mathrm{RT}$ spray model constants characterizing the breakup time and length scales was conducted through comparison of predicted SMD profiles against USAXS measurements. The optimal spray model constants resulted in good agreement across a range of injection pressures $(50-150 \mathrm{MPa})$ and ambient densities $\left(7.6-22.8 \mathrm{~kg} / \mathrm{m}^{3}\right)$ for the ECN Spray A injector, with a nozzle diameter of approximately $90 \mu \mathrm{m}$ and K-factor of 1.5. Application of these model constants for the Spray D simulation resulted in excellent agreement with the measured SMD profile along the spray centerline, as shown in Figure 8(b). These results indicate that the response of the centerline SMD profile to changes in injection pressure, ambient density and injector nozzle diameter can be well captured by the KH-RT aerodynamic breakup spray model. Although this model is very simple in comparison to higher fidelity modeling approaches, $\mathrm{x}$-ray data enabled the unique characterization and assessment of an efficient modeling approach to represent the salient features of the spray. This comparison among the models highlights the potential of not only assessing and validating engineering models against USAXS measurements, but also against higher fidelity modeling approaches to gain insight into the characteristic length and time scales characterizing unobservable spray processes influencing the overall spray structure.

The final details of the predicted spray are assessed by comparing predicted and measured transverse SMD profiles, as shown in Figure 
9 at axial positions of (a) $2.0 \mathrm{~mm}$ and (b) $10.0 \mathrm{~mm}$. At an axial position of the $2.0 \mathrm{~mm}$, the range of predicted SMD along the spray centerline is between 42-68 $\mu \mathrm{m}$. In general, the Lagrangian and VOF-LES models, which consider the contribution of both the liquid core and detached droplets to the SMD, predict similar SMD profiles across the width of the spray. The SMD values along the periphery closely match those predicted by the CLSVOF model for detached droplets. The droplet sizes predicted by the VOF methods in the periphery of the transverse profiles compared to the measurements provides an indication of the resolution that is still needed by both models to achieve optimal agreement. Indeed, with a minimum grid resolution of $2.5 \mu \mathrm{m}$, the VOF-LES approach overpredicts the measured SMD profile, while the CLSVOF approach with a minimum grid resolution of $1.7 \mu \mathrm{m}$ more closely matches the peripheral SMD. The CLSVOF predictions highlight the contribution of detached droplets to the predicted SMD, and suggest that the size of formed droplets is relatively constant across the width of the spray.

The Eulerian $\Sigma$-Y model is seen to predict a much more aggressive atomization process than is predicted by the other models, with $75 \%$ of the spray width comprised of SMD values less than $1 \mu \mathrm{m}$. For the Spray A injector, USAXS measurements of SMD indicated values generally greater than $1 \mu \mathrm{m}$ [22]. With Spray D having a larger injector nozzle than Spray A, it is therefore less likely for sub-micron droplets to exist in the spray. Careful calibration of the Eulerian $\Sigma$-Y model would allow for improved prediction of droplets formed from the atomization process. Indeed, as noted by Pandal and co-workers [56], careful calibration of the constants characterizing the turbulent mixing time scale and equilibrium radius against the detailed USAXS projected surface area measurements can yield improved predictions for the evolution of the predicted surface area density. Because the Eulerian $\Sigma$-Y model predicted projected mass (and therefore projected volume) distributions that were in agreement with the experimental data as previously shown in Figure 5(b), improved predictions of the projected surface area in the near-nozzle region would likely yield improved predictions for the transverse SMD profile.

At an axial position of $10.0 \mathrm{~mm}$, the Lagrangian and Eulerian $\Sigma$-Y model predictions are compared with the SMD measurements. Although good agreement is seen among the model predictions and experimental data at the spray centerline, the models predict different profile shapes. While the experimental data suggests that a peak in the SMD distribution can be found at the spray centerline, local minima can be found at locations away from the spray centerline. While the Eulerian $\Sigma$-Y model predicts the correct shape, the droplet sizes are generally underpredicted and the spray width is overpredicted. Although the SMD profile predicted by the Lagrangian spray model indicates spatial fluctuations of approximately $1 \mu \mathrm{m}$, these fluctuations are likely due to the sampling on the computational parcels on a fine post-processing grid and should be reduced by increasing the sampling frequency of the data during the steady portion of injection. Even with this existing predicted profile, the Lagrangian model predicts a relatively constant droplet size distribution in the central region of the spray, with a slight increase along the spray periphery. The different shapes in the SMD profiles predicted by the Lagrangian and Eulerian $\Sigma$-Y models may be due to differences in droplet collision modeling. In the Lagrangian model, the influence of droplet collisions on the resultant spray structure is neglected, whereas the influence of droplet collisions is accounted for in the Eulerian model, as previously described in Equation (4). Future computational investigations can explore the influence of droplet collisions on the transverse SMD profiles in the Lagrangian modeling framework to ensure better representation of the spray structure.

Page 11 of 14

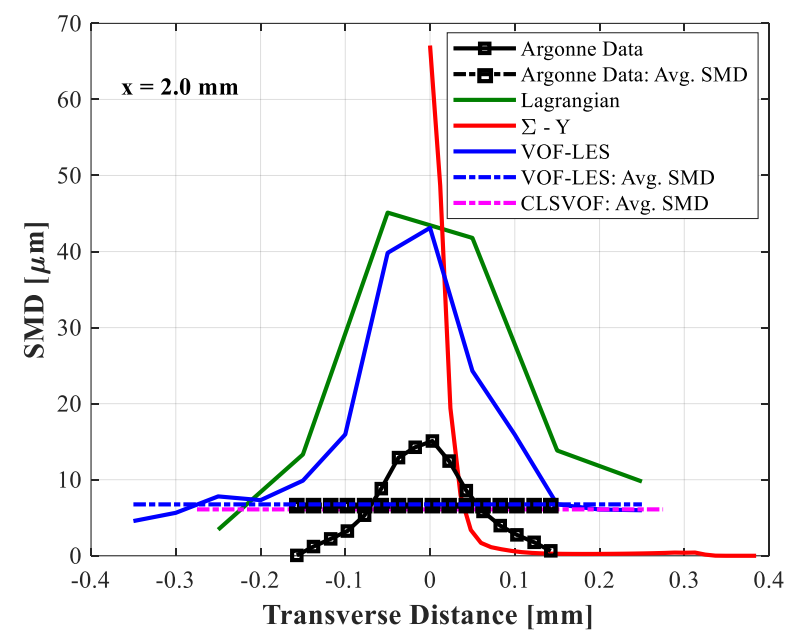

(a)

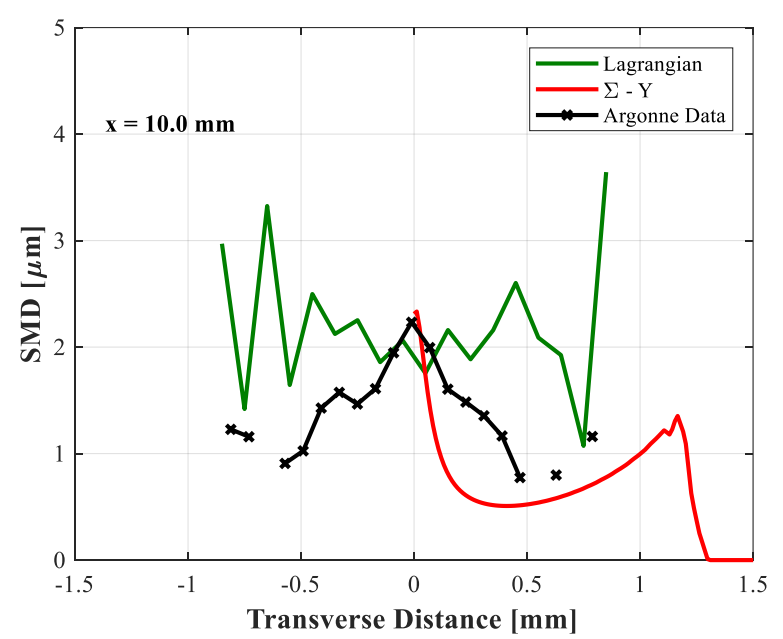

(b)

Figure 9. Comparison of measured and predicted transverse SMD distributions at axial distances of (a) $2.0 \mathrm{~mm}$ and (b) $10.0 \mathrm{~mm}$ from the nozzle exit. Solid and dashed lines are used to denote transverse and spatially-averaged SMD, respectively.

\section{SUMMARY/CONCLUSION}

This work has reviewed and compared experimental and simulated primary atomization data conducted for the Engine Combustion Network (ECN) Spray D injector, and presented at recently held $5^{\text {th }}$ workshop of the ECN. Through physically consistent comparisons between the measured and predicted spray quantities, improved understanding of the spray structure in the near-nozzle region was achieved. The major findings from this joint experimental and modeling campaign are summarized below:

1. Traditional Lagrangian approaches are not able to capture the mass distribution in dense regions of the spray. In spite of this deficiency, the Lagrangian model is able to reproduce many of the features of the spray, particularly the centerline SMD distribution. This is only possible through careful calibration of the KH-RT spray breakup model constants against the x-ray data. Previous studies revealed the ability of the aerodynamic-induced breakup model to capture the experimentally observed sensitivities of the centerline SMD distribution to changes in injection pressure and ambient 
density. The comparison presented in this paper now validates the ability of the KH-RT model to capture the sensitivity of the centerline SMD to changes in nozzle diameter.

2. The Eulerian $\Sigma$-Y approach works quite well in predicting the dense spray mass distribution features in the near-nozzle region. However, this approach still relies on model tuning over a range of conditions for droplet diameter and surface area density predictions. Therefore, surface area models, which rely on mass distribution could yield misleading results if mass distribution in dilute regions of the spray cannot be adequately captured. Because these shortcomings of the models are typically compensated through model tuning, the Ultra Small Angle X-ray Scattering (USAXS) measurements offer a unique opportunity for direct comparison with predicted surface area density evolution to allow for informed calibration studies.

3. A model-free approach to resolving the spray structure based on interface capturing, as utilized in the LES-VOF and CLSVOF methods, offers an opportunity to explore the details of the predicted spray in the near-nozzle region. Simulation results revealed that formation of droplets from the primary atomization process is delayed until distances beyond $1 \mathrm{~mm}$ from the injector nozzle exit. Droplet sizes formed from the primary atomization process are predicted to be relatively constant across the width of the spray in the near-nozzle region.

4. Some of the differences between the LES-VOF and CLSVOF results can be directly attributed to the different grid densities afforded in the two simulations, particularly in the capability of resolving surface corrugation, ligament necking and break-up. More subtle differences might be due to the adoption of the LES framework in VOF-LES with respect to the no-model approach adopted for turbulence in CLSVOF. Future work might be able to elucidate the advantage brought by LES in this application domain.

5. Comparison between experimentally measured and predicted interfacial area profiles reveals the potential of the USAXS measurement to distinguish regions of continuous liquid structures and finely atomized droplets. In central regions of the spray, the projected surface area profile may be related to the degree of atomization of the liquid jet; local minima may indicate regions of poorly atomized spray comprised of larger liquid structures and detached droplets, whereas local maxima may indicate regions of completely atomized spray.

Overall the X-ray radiography and USAXS data provide a unique new dataset for understanding characteristics of the spray, particularly in the dense near-nozzle region. Future investigations should evaluate how the highly resolved scientific spray modeling results, such as those produced by the VOF-LES and CLSVOF results can be used to improve spray sub-models for engineering-level spray simulations, such as turbulent-dispersion and droplet-droplet collisions.

\section{REFERENCES}

1. Kalghatgi, G. T. (2015). Developments in internal combustion engines and implications for combustion science and future transport fuels. Proc. Combust. Inst., 35, 101-115.

2. Chadwell, C., Alger, T., Roberts, C., Arnold, S. (2011). Boosting simulation of high efficiency alternative combustion mode engines. SAE Technical Paper 2011-01-0358.
3. Manente, V., Johansson, B., Cannella, W. (2011). Gasoline partially premixed combustion, the future of internal combustion engines? Int. J. Engine Research, 12, 194-208.

4. Shinjo J. and Umemura A., "Detailed simulation of primary atomization mechanisms in Diesel jet sprays (isolated identification of liquid jet tip effects)," Proc. Combust. Inst., vol. 33, no. 2, pp. 2089-2097, 2011.

5. Marmottant, P., Villermaux, E. (2004). On spray formation. Journal of Fluid Mechanics, 498, 73-112.

6. Deshpande S. S., Gurjar S. R., Trujillo M. F., A computational study of an atomizing liquid sheet, Physics of Fluids 27, 082108 (2015).

7. Gorokhovski, M. and Herrmann, M., "Modeling Primary Atomization," Annual Review of Fluid Mechanics, 2008.

8. Desjardins, O. and Pitsch, H., "Detailed numerical investigation of turbulent atomization of liquid jets," Atomization and Sprays, 20(4): 311-336, 2010.

9. Jemison, M., Sussman, M., and Arienti, M., "Compressible, Multi-Phase Semi-Implicit Method with Moment of Fluid Interface Representation.” J. of Computational Physics, 279, 182217, 2014.

10. Tryggvason, G., Scardovelli, R. and Zaleski, S., Direct Numerical Simulations of Gas-Liquid Multiphase Flows, Cambridge University Press, 2011.

11. Vallet, A., Burluka, A., and Borghi, R., "Development of a Eulerian model for the atomization of a liquid jet," Atomization Sprays, vol. 11, pp. 619-642, 2001.

12. Garcia-Oliver, J. M., Pastor, J. M. and Pandal, M. (2013). Diesel spray CFD simulations based on the $\Sigma$-Y eulerian atomization. Atomization and Sprays 23, 1.

13. Payri, R., Gimeno, J., Martí-Aldaraví, P., Alarcón, M., “A new approach to compute temperature in a liquid-gas mixture. Application to study the effect of wall nozzle temperature on a Diesel injector," International Journal of Heat and Fluid Flow, 68:79-86, 2017.

14. Subramaniam, S., "Lagrangian-Eulerian methods for multiphase flows," Prog. Energy Combust. Sci, vol. 39, pp. 215-245, 2013.

15. Dukowicz, J. K., "A particle-fluid numerical model for liquid sprays," Journal of Computational Physics, 35(2): 229-253, 1980.

16. Bachalo, W.D., "Spray Diagnostics for the twenty-first century," Atomization and Sprays, 3:439-474, 2000.

17. Dumouchel, C., "On the experimental investigation on primary atomization of liquid streams," Exp. Fluids, 45:371-422, 2008.

18. Fansler, T.D., Parrish, S.E., "Spray measurement technology: a review," Measurement Science and Technology, 2014.

19. Zaheer, H., "Transient Microscopy of Primary Atomization in Gasoline Direct Injection Sprays,” M.S. Thesis, Georgia Institute of Technology, 2015.

20. J.Wang, "X-ray vision of fuel sprays," Journal of synchrotron radiation, vol. 12, no. 2, pp. 197-207, 2005.

21. Y. Yue, C. F. Powell, R. Poola, J. Wang, and J. K. Schaller, "Quantitative measurements of diesel fuel spray characteristics in the near-nozzle region using x-ray absorption," Atomization and Sprays, vol. 11, no. 4, 2001.

22. Kastengren, A., Ilavsky, J., Viera, J.P., Payri, R., Duke, D.J., Swantek, A., Tilocco, F.Z., Sovis, N., Powell, C.P. 2017. Measurements of droplet size in shear-driven atomization using ultra-small angle x-ray scattering. Int. J. Multi. Flow 92, 131-139.

23. Engine Combustion Network, "Engine Combustion Network Experimental Data Archive," https://ecn.sandia.gov, accessed Oct. 2017.

24. Payri, R., Gimeno, J., Cuisano, J., and Arco, J., "Hydraulic characterization of diesel engine single-hole injectors," Fuel, 180: 357-366, 2016.

Page 12 of 14 
25. Magnotti, G.M and Genzale, C.L., "Exploration of Turbulent Atomization Mechanisms for Diesel Spray Simulations," SAE Technical Paper 2017-01-0829, 2017.

26. Kastengren, A.L., Powell, C.F., Riedel T., Cheong, S.-K., Im, K.S., Liu, X. Wang, Y.J., and Wang, J., "Nozzle geometry and injection duration effects on diesel sprays measured by X-ray radiography," J. Fluid. Eng., 2008.

27. A. Kastengren, C.F. Powell, D. Arms, E.M. Dufresne, H. Gibson, and J. Wang., "The 7BM beamline at the APS: a facility for timeresolved fluid dynamics measurements," J Synchrotron Rad, 19:654-657, 2012.

28. Ilavsky, J., Jemian, P., Allen, A., Zhang, F., Levine, L., Long, G., 2009. Ultra-small-angle $x$-ray scattering at the Advanced Photon Source. J. Appl. Cryst. 42, 469-479.

29. Bonse, U., Hart, M., 1965. Tailless x-ray single-crystal reflection curves obtained by multiple reflection. Appl. Phys. Lett. 7, 238 240

30. Ilavsky, J., Jemian, P., 2009. Irena: tool suite for modeling and analysis of small-angle scattering. J. Appl. Cryst. 42, 347-353.

31. Ilavsky, J., Zhang, F., Allen, A.J., Levine, L.E., Jemian, P.R., Long, G.G., 2013. Ultra-small-angle X-ray scattering instrument at the advanced photon source: history, recent development, and current status. Metallurgical and Mater. Trans. A 44A(c1), 68-76.

32. Narayanan, T., 2007. Synchrotron small-angle x-ray scattering. In: Borsali, R., Pecora, R. (Eds.), Soft Matter: Scattering, Imaging, and Manipulation editors. Springer, Dordrecht.

33. Magnotti, G.M., "Modeling the influence of nozzle-generated turbulence on the diesel sprays," $\mathrm{PhD}$ Thesis, Georgia Institute of Technology, 2017.

34. Magnotti, G.M. and Genzale, C.L., "Detailed assessment of diesel spray atomization models using visible and X-ray extinction measurements," International Journal of Multiphase Flows, 97:33-45, 2017.

35. Senecal, P.K., Pomraning, E., Richards, K.J., Som, S., "GridConvergent Spray Models for Internal Combustion Engine Computational Fluid Dynamics Simulations," Journal of Energy Resources Technology, 136(1): 2013.

36. Magnotti, G.M., Matusik, K.E., Duke, D.J., Knox, B.W., Martinez, G.L., C.F. Powell, A.L. Kastengren, and Genzale, C.L., "Modeling the Influence of Nozzle-Generated Turbulence on Diesel Sprays," ILASS Americas 29th Annual Conference, Atlanta, GA, May 2017.

37. Beale J. C. and Reitz R. D., "Modeling Spray Atomization with the Kelvin-Helmholtz/Rayleigh-Taylor Hybrid Model," Atomization and Sprays, 9:623-650, 1999.

38. Han, Z., and Reitz R.D., "Turbulence modeling of internal combustion engines using RNG k-e models," Comb. Sci. Tech., 106:267-295, 1995.

39. Pope, S., "An explanation of the turbulent round-jet/plane-jet anomaly," AIAA Journal, 16:279-281, 1978.

40. Desantes, J.M., Payri, R., Gimeno, J., Martí-Aldaraví, P., "Simulation of the first millimeters of the diesel spray by an Eulerian spray atomization model applied on ECN Spray A injector," SAE Technical Paper 2014-01-1418, 2014.

41. Senecal, P., Richards, K., Pomraning, E., and Yang, T. "A New Parallel Cut-Cell Cartesian CFD Code for Rapid Grid Generation Applied to in-Cylinder Diesel Engine Simulations." SAE Technical Paper 2007-01-0159, 2007.

42. Brackbill J.U., Kothe D.B., Zemach C.A., "A Continuum Method for Modeling Surface Tension," Journal of Computational Physics, 100(2), 335-354, 1992.

43. Gueyffier D., Li J., Nadim A., Scardovelli R. and Zaleski S., Volume-of-Fluid Interface Tracking with Smoothed Surface Stress Methods for Three-Dimensional Flows. J. Comput. Phys. 152, 2 (1999), 423-456.

Page 13 of 14
44. Rutland, C.J., Large-eddy simulations for internal combustion engines - a review. International Journal of Engine Research, 2011. 12(5): p. 421-451.

45. Befrui, B., Corbinelli, G., Robart, D., Reckers, W. et al., "LES Simulation of the Internal Flow and Near-Field Spray Structure of an Outward-Opening GDi Injector and Comparison with Imaging Data," SAE Technical Paper 2008-01-0137, 2008.

46. Battistoni, M., Duke, J. D., Swantek, A. L., Powell, F. Z., and Som, S. "The Effect of Non-Condensable Gas on Cavitating Nozzles." Atomization and Sprays 25 (2015).

47. Xue, Q., Battistoni, M., Powell, C.F., Longman, D.E., Quan, S., Pomraning, E., Senecal, P.K., Schmidt, D.P., and Som, S., "An Eulerian CFD Model and X-ray Radiography for Coupled Nozzle Flow and Spray in Internal Combustion Engines." International Journal of Multi-Phase Flows 70 (2015): 77-88.

48. Zhao, H., Quan, S., Dai, M., Pomraning, E., Senecal, P.K., Xue, Q., Battistoni, M., and Som, S. "Validation of Three-Dimensional Internal Nozzle Flow Including Automatic Mesh Generation and Cavitation Effects." ASME J. Eng. Gas Turbines Power, 2014.

49. Matusik K. E., Duke D. J., Kastengren A. L., Sovis N., Swantek A. B. and Powell C. F., "High-resolution X-ray tomography of Engine Combustion Network diesel injectors," Int. J. Engine Res, in press. Accepted 09/22/2017.

50. Kwatra, N., Su, J., Grétarsson, J.T., and Fedkiw, R. (2009) “A method for avoiding the acoustic time step restriction in compressible flow." J. Comp. Phys. 228: 4146-4161.

51. Arienti, M. and Sussman, M., "A Numerical Study of the Thermal Transient in High-Pressure Diesel Injection.” Int. J. of Multiphase Flow 88: 205-221 (2017).

52. Sussman, M., and Ohta, M. "A Stable and Efficient Method for Treating Surface Tension in Incompressible Two-phase Flow". SIAM J. Sci. Comput., 31(4):2447-2471, 2009.

53. Arienti, M. and Sussman, M., "An embedded level set method for sharp-interface multiphase simulations of Diesel injectors," International Journal of Multiphase Flow, 59:1-14, 2014.

54. Kastengren, A.L., Powell, C.F, Wang, Y., Im, K.-S., Wang, J., "X-ray Radiography Measurements of Diesel Spray Structure at Engine-like Ambient Density," Atomization and Sprays, 19(11): 1031-1044, 2009.

55. Magnotti, G.M., and Genzale, C.L., "A Novel Approach to Assess Diesel Spray Models using Joint Visible and X-Ray Liquid Extinction Measurements," SAE Int. J. Fuels Lubr. 8(1):167-178, 2015.

56. Pandal, A., Pastor, J.M., Payri, R., Kastengren, A. et al., "Computational and Experimental Investigation of Interfacial Area in Near-Field Diesel Spray Simulation," SAE Int. J. Fuels Lubr., 10(2):423-431, 2017.

\section{CONTACT INFORMATION}

Prof. Michele Battistoni

michele.battistoni@unipg.it

Dr. Gina M. Magnotti

gmagnotti@anl.gov

\section{ACKNOWLEDGMENTS}

The following individuals and funding agencies are acknowledged for their support.

Università degli Studi di Perugia: We acknowledge the computational resources provided by the Italian Cineca consortium, under the Iscra 
initiative - awards HP10CYWGHT,2016 and HP10C776NM,2017 on the HPC cluster Galileo and supercomputer Marconi.

Georgia Institute of Technology: This material is based upon work supported jointly by the Department of Energy, Office of Energy Efficiency and Renewable Energy (EERE), and the Department of Defense (DOD) under Award Number DE-EE0073333. We also gratefully acknowledge the computing resources provided on Blues, a high-performance computing cluster operated by the Laboratory Computing Resource Center at Argonne National Laboratory.

Argonne National Laboratory: Experiments were performed at the $7-$ BM and 9-ID beamlines of the APS at Argonne National Laboratory.
Use of the APS is supported by the U.S. Department of Energy (DOE) under Contract No. DE-AC02-06CH11357. Argonne's x-ray research of Advanced Combustion is funded by the DOE Office of Energy Efficiency and Renewable Energy Vehicle Technologies Office under the direction of Gurpreet Singh and Leo Breton.

Sandia National Laboratories: Work was supported by the Laboratory Directed Research and Development program at Sandia. Sandia National Laboratories is a multimission laboratory managed and operated by National Technology and Engineering Solutions of Sandia, LLC., a wholly owned subsidiary of Honeywell International, Inc., for the U.S. Department of Energy's National Nuclear Security Administration under contract DE-NA-0003525. 